\title{
Study of the Visceral Adipose Tissue in a Cohort of Patients with Moderate-Severe Psoriasis Treated with Biological Therapy
}

\author{
Ruiz-Villaverde Ricardo, Ruiz-Carrascosa José C
}

1 Dermatology Department, Hospital Universitario San Cecilio, Granada, Spain

Key words: psoriasis, biological therapy, visceral adipose tissue, treatment, severity.

Citation: Ruiz-Villaverde R, Ruiz-Carrascosa JC. Study of the visceral adipose tissue in a cohort of patients with moderate-severe psoriasis treated with biological therapy. Dermatol Pract Concept. 2022;12(1):e2022032. DOI: https://doi.org/10.5826/dpc.1201a32

Accepted: August 21, 2021; Published: January 2022

Copyright: (02022 Ruiz-Villaverde and Ruiz-Carrascosa. This is an open-access article distributed under the terms of the Creative Commons Attribution-NonCommercial License (BY-NC-4.0), https://creativecommons.org/licenses/by-nc/4.0/, which permits unrestricted noncommercial use, distribution, and reproduction in any medium, provided the original authors and source are credited.

Funding: None.

Competing interests: None.

Authorship: Both authors have contributed significantly to this publication.

Corresponding author: Ricardo Ruiz-Villaverde. Servicio de Dermatología. Hospital Universitario San Cecilio, Granada, Spain. E-mail: ismenios2005@gmail.com

\footnotetext{
ABSTRACT Introduction: Visceral adipose tissue (VAT) has a greater relationship with the genesis of the metabolic syndrome and the pathology associated with obesity.

Methods: A cross-sectional study of patients with moderate-severe psoriasis in the Psoriasis Unit of the San Cecilio University Hospital in Granada in the period July 1, 2020 - December 31, 2020, was performed. All the patients $(\mathrm{n}=110)$ were receiving biological therapy to control the disease. The variables measured included age, sex, time since diagnosis, weight, height, body mass index (BMI), visceral and total fat, and severity parameters. The visceral fat index was evaluated using a bioimpedance scale, considering a cut-off point for a healthy level $<12$.

Results: Our sample consisted of 110 patients with a mean age of 47.47 years, with a clear predominance of males $(61.7 \%$ of patients). After testing for normality using the Kolmogorov-Smirnov test, the Mann-Whitney U test for nonparametric data for independent samples was used, which revealed significant differences between the number of previous treatments and visceral fat $(\mathrm{U}=-2.235, \mathrm{P}=$ 0.025 ). No statistically significant differences were found when correlating total fat or visceral fat with BMI.

Conclusions: The results presented lead us to consider if the levels of VAT could be a factor that contributes to some extent to therapeutic refractoriness. The determination of VAT using bioimpedance scales in patients with moderate-severe psoriasis is a valuable method to measure VAT.
} 


\section{Introduction}

Adipose tissue generally considered a useful annex to provide protection, heat, and energy, has surpassed these activities, and as its study progresses it has positioned itself as an organ with neuroimmune-endocrine functions. Through the production of molecules such as hormones, antimicrobials, cytokines, and adipokines, it participates in the function of various cells and organs, which allows it to intervene in the defense and homeostasis of the body. Fat deposits are different from each other, even between those of the same type of adipose tissue. Each one is complex, made up of different cells, with different functions and variations both in gene expression and in their response to hormones (the subcutaneous of the thighs responds to sex hormones, the neck, upper back and abdomen to corticosteroids) [1].

Visceral adipose tissue (VAT) is divided into omental or epiploic and mesenteric. It occupies the spaces between the abdominal organs and keeps them in place. It has lymph nodes and a greater number of blood vessels and adrenergic receptors than the rest of the white adipose tissue. Adipocytes in visceral tissue also express a greater number of receptors for corticosteroids, and in obesity, the enzyme 11 beta-hydroxysteroid dehydrogenase is overexpressed, which generates active substances from inactive glucocorticoids, stimulating adipogenesis and increase visceral fat. Therefore, visceral adipose tissue has a greater relationship with the genesis of the metabolic syndrome and the pathology associated with obesity $[2,3]$.

The most accurate way to measure visceral fat level is with a computerized tomography (CT) or magnetic resonance imaging (MRI) scan. But without doubt, the most practical and simple way that will allow us to also measure many other parameters with greater or lesser precision depending on their quality is through a bioimpedance scale. Our body is normally made up of $50 \%$ to $70 \%$ water. Fat tissue barely has $10 \%$ so it is a worse conductor, generating resistance to the passage of electricity. This fact is used by bioimpedance scales to measure the time it takes for the electricity to pass through our body. Considering our weight, age and race, the percentage of body fat, visceral and bone density may be calculated.

\section{Objectives}

- To know the total and average visceral fat composition in a cohort of patients with moderate-severe psoriasis treated with biological therapy in a tertiary hospital using a biompedance scale.

- Establish the correlation of visceral adipose tissue with the body mass index (BMI) of the patients that make up this cohort.

- Establish if there is a relationship with the number of treatments and, therefore, refractoriness to them in patients with moderate-severe psoriasis.

\section{Methods}

A cross-sectional study of patients with moderate-severe psoriasis in the Psoriasis Unit of the San Cecilio University Hospital in Granada in the period July 1, 2020 - December 31, 2020 was performed. Patients were obtained by consecutive sampling. All patients signed an informed consent that they could revoke at any time during the study. The study is approved by the Ethics Committee of our hospital with code HUSC-DER-005.

The diagnosis of moderate-severe psoriasis was established when the patient met the following requirements (Psoriasis Area Severity Index [PASI] > 10, Body Surface Area [BSA] > 10 and / or Dermatology Life Quality Index [DLQI] $>10)$. All the patients were receiving biological therapy to control the disease.

The variables measured included age, sex, time since diagnosis, weight, height, BMI, visceral and total fat, and severity parameters (PASI, BSA, DLQI). All previous treatments that the patient had received, including systemic treatments and biological treatments, were considered. Treatment with biologically effective UVB phototherapy was excluded as a treatment to consider. Only the overall number of treatments was considered in those patients where the cause of switching was a primary or secondary failure, excluding safety reasons.

The visceral fat index was evaluated using a bioimpedance scale (Kanthor $\left.{ }^{\circledR}\right)$, considering a cutoff point for a healthy level $<12[4,5]$.

The statistical software IMB SPSS version 25 in its macOS version was used for all analyses. The Kolmogorov Smirnov test was used to test the normality of the data. Measures of central tendency and dispersion were used depending on the nature of the data. Outcome variables were selected according to clinical criteria. The visceral fat variable was categorized according to the healthy cutoff point $<12$ in a binomial variable for the analyses. Spearman correlation test for non-parametric samples as well as the Mann-Whitney U test for independent samples were used to study the association and relationship between the variables collected. A statistical significance level of $\mathrm{P}<0.05$ was set.

\section{Results}

Our sample consisted of 110 patients with a mean age of 47.47 years, with a clear predominance of males $(61.7 \%$ of patients). The whole sample was placed to the right of the cutoff point considered as pathological in relation to the determination of visceral fat. The rest of the characteristics evaluated are reported in Table 1.

After testing for normality using the Kolmogorov-Smirnov test, the Mann-Whitney U Test for nonparametric data for independent samples was used, which revealed significant 
Table 1. Clinical and anthropometric characteristics of the participants.

\begin{tabular}{|l|l|}
\multicolumn{1}{c|}{ Outcome } & \multicolumn{1}{c|}{ Values } \\
\hline Age & $47.47 \pm 13.47$ yearss \\
\hline Gender & $74(61.7)$ \\
\hline \multicolumn{1}{|c|}{ Male } & $46(38.3)$ \\
\hline \multicolumn{1}{|c|}{ Female } & $22.84 \pm 12.81$ years \\
\hline $\begin{array}{l}\text { Time since diagnosis } \\
\text { (years) }\end{array}$ & $82.79 \pm 21.00$ \\
\hline Weight $(\mathrm{kg})$ & $1.70 \pm 0.1$ \\
\hline Height $(\mathrm{m})$ & $28.49 \pm 6.10$ \\
\hline BMI & $11.97 \pm 7.30$ \\
\hline Visceral Fat & $29.02 \pm 11.36$ \\
\hline Total Fat & $12.93 \pm 5.23$ \\
\hline PASI & $15.18 \pm 7.42$ \\
\hline BSA & $11.72 \pm 4.66$ \\
\hline DLQI &
\end{tabular}

Data are presented as mean \pm standard deviation or $\mathrm{n}(\%)$, as appropriate.

differences between the number of pre-treatments and visceral fat: $\mathrm{U}=-2.235 ; \mathrm{P}=0.025)$. These results showed a positive correlation between high levels of visceral fat with a greater number of previous treatments. No statistically significant differences were found when correlating total fat or visceral fat with BMI (Table 2).

\section{Conclusions}

Psoriasis is a chronic inflammatory disease that is associated with a high degree of systemic and vascular inflammation. It is known to cause an increase in cardiovascular (CV) risk factors, coronary artery disease and myocardial infarction. VAT is associated with this vascular inflammation and CV events, which have characteristically been associated with elevated BMI [4].

Although BMI is a clinical marker of obesity, this obesity is an heterogeneous condition with varying CV and metabolic manifestations [6]. Obesity causes increased levels of proinflammatory cytokines (TNF $\alpha$ and IL6). High levels of TNF $\alpha$ would produce a reduction in adiponectin levels, which would lower HDL cholesterol levels, increase triglycerides and insulin resistance. This leads to an increase in VAT and IL6 levels that perpetuate the inflammatory process [5]. In our study, it is shown in a cohort of 110 patients treated with biological therapy that the mean is right at the cutoff point considered pathological for visceral fat levels (11.97 +/- 7.30; VAT > 12) and we reported a statistical correlation with the number of previous treatments the patient had received. This makes us wonder if these levels could be a factor that contributes to some extent to therapeutic refractoriness. We were unable to find statistically significant differences when trying to establish a correlation between BMI levels and visceral fat levels. Interestingly, the mean BMI of the presented cohort does not exceed 30 points that mark the limit of obesity, but there is a clear overweight, as well as the levels of visceral fat that have not reached the limit considered as pathological.

Different techniques (mainly imaging) have been postulated to determine VAT. Coronary CT angiography is a non-invasive imaging technique, of great prognostic utility and a predictor of CV events. Sajja et al have shown in a one-year study that the reduction in VAT volume measured by this technique is associated with an improvement in the coronary artery burden [7].

Other techniques include ultrasonography (USG), which is regarded as a radiation technique with a lower cost than the one of CT [8]. USG measures the maximal thickness of preperitoneal mass at the anterior surface of the liver and the minimal thickness of subcutaneous fat in the upper median abdomen. Gönul et al performed the first study using this technique, in which they concluded a higher prevalence of

Table 2. Bivariate correlations between number of previously treatments and anthropometric variables.

\begin{tabular}{|c|c|c|c|c|c|c|}
\hline & Age & Gender & BMI & Total Fat & $\begin{array}{c}\text { Categorised Visceral } \\
\text { Fat }\end{array}$ & $\begin{array}{l}\text { Number of } \\
\text { previously } \\
\text { treatments }\end{array}$ \\
\hline Age & & 0,015 & $0.411 \%$ & $0.180 *$ & 0.087 & -0.023 \\
\hline Gender & 0,015 & & $-0.231 *$ & $-0.392 * *$ & $-0.433 * *$ & 0.119 \\
\hline BMI & $0.411 * *$ & $-0.231 *$ & & $0.691 * *$ & $0.629 * *$ & -0.059 \\
\hline Total Fat & $0.180 *$ & $-0.392 * *$ & $-691 \%$ & & $0.733 *$ & -0.158 \\
\hline Categorised visceral fat & 0,087 & $-0.433 *$ & $0-629 * *$ & $0.733 *$ & & $-0.205 *$ \\
\hline $\begin{array}{l}\text { Number of previously } \\
\text { treatments }\end{array}$ & $-0,023$ & 0.119 & -0.059 & -0.158 & $-0.205 \%$ & \\
\hline
\end{tabular}

Spearman correlation coefficient. "Correlation is significant at the 0.05 level; * Correlation is significant at the 0.01 level. 
metabolic syndrome in patients with psoriasis but with an abdominal fat index similar to the control group [9]. As in our study, in this case with an imaging technique, the authors were unable to establish a relationship between visceral fat levels and other markers related to metabolic syndrome.

Finally, studies have also been conducted using 18F-Fluorodeoxyglucose positron-emission tomography to measure VAT volume and its relationship with vascular inflammation in patients with psoriasis. It shows how new treatments for moderate-severe psoriasis lower the volume of VAT and vascular inflammation, which is why VAT is an important CV biomarker [10].

Our study has some limitations. It is a cross-sectional study, which has measured the visceral fat of our patients at a specific and determined moment. Although there is indeed a statistically significant correlation between the number of previous biological treatments (used due to refractoriness to treatment) and the levels of visceral fat, we have not been able to detect an association with other characteristics that have been used as biomarkers and prognoses factors in metabolic syndrome such as BMI. Furthermore, we have not been able to establish a correlation with imaging techniques already used in other studies due to the lack of availability of these techniques in our centre.

As a conclusion to our study, we want to value the determination of VAT using bioimpedance scales in patients with moderate-severe psoriasis. Further studies are required to determine its correlation with other characteristics of the disease and its positioning in relation to its determination using other imaging techniques already discussed.

\section{References}

1. Vega-Robledo GB, Rico-Rosillo MG. Adipose tissue: Immune function and alterations caused by obesity. Rev Alerg Mex. 2019;66(3):340-353. Spanish. DOI: 10.29262/ram.v66i3.589. PMID: 31606018.
2. Rehrer CW, Karimpour-Fard A, Hernandez TL, et al. Regional differences in subcutaneous adipose tissue gene expression. Obesity (Silver Spring). 2012;20(11):2168-2173. DOI: 10.1038/ oby.2012.117. PMID: 22627919; PMCID: PMC3434286.

3. Vohl MC, Sladek R, Robitaille J, et al. A survey of genes differentially expressed in subcutaneous and visceral adipose tissue in men. Obes Res. 2004;12(8):1217-1222. DOI: 10.1038/ oby.2004.153. PMID: 15340102.

4. Toussirot E, Aubin F, Desmarets M, et al. Visceral adiposity in patients with psoriatic arthritis and psoriasis alone and its relationship with metabolic and cardiovascular risk. Rheumatology (Oxford). 2021;60(6):2816-2825. DOI: 10.1093/rheumatology/ keaa720. PMID: 33232483.

5. Goolam Mahyoodeen N, Crowther NJ, Pillay L, et al. Relationship of visceral fat and adipokines with cardiometabolic diseases in psoriasis. Acta Derm Venereol. 2019; 99:1218-1223. DOI: 10.2340/00015555-3327. PMID: 31580467.

6. Budu-Aggrey A, Brumpton B, Tyrrell J, et al. Evidence of a causal relationship between body mass index and psoriasis: A mendelian randomization study. PLoS Med. 2019;16(1):e1002739. DOI:10.1371/journal.pmed.1002739. PMID: 30703100; PMCID: PMC6354959.

7. Sajja A, Abdelrahman KM, Reddy AS, et al. Chronic inflammation in psoriasis promotes visceral adiposity associated with noncalcified coronary burden over time. JCI Insight. 2020;5(22):e142534. DOI: 10.1172/jci.insight.142534. PMID: 33104056; PMCID: PMC7710282.

8. Balci A, Balci DD, Yonden Z, et al. Increased amount of visceral fat in patients with psoriasis contributes to metabolic syndrome. Dermatology. 2010;220(1):32-37. DOI: 10.1159/000254482. PMID: 19887761.

9. Gönül M, Tatar İ, Canpolat F, Işı1 Kurmus G, Ergin C, Hekimoğlu B. Evaluation of abdominal fat index by ultrasonography and its relationship with psoriasis and metabolic syndrome. Postepy Dermatol Alergol. 2017;34(5):453-456. DOI: 10.5114/ ada.2017.71111. PMID: 29507560. PMCID: PMC5831280..

10. Rivers JP, Powell-Wiley TM, Dey AK, et al. Visceral Adiposity in Psoriasis is Associated With Vascular Inflammation by $18 \mathrm{~F}-\mathrm{Flu}-$ orodeoxyglucose Positron-Emission Tomography/Computed Tomography Beyond Cardiometabolic Disease Risk Factors in an Observational Cohort Study. JACC Cardiovasc Imaging. 2018;11(2 Pt 2):349-357. DOI: 10.1016/j.jcmg.2017.08.014. PMID: 29055628. PMCID: PMC5803350. 